\title{
Gut Microbiota Signatures in Gestational Anemia
}

\author{
Yan Long ${ }^{1 \dagger}$, Fang Liang ${ }^{2 \dagger}$, Ruochun Guo ${ }^{2 \dagger}$, Chunyan Zhu ${ }^{3}$, Xueqin Zhao ${ }^{1}$, Xifan Wang ${ }^{2}$, \\ Fei Liu ${ }^{1}$, Min Jiang ${ }^{1}$, Qihua Liang ${ }^{1}$, Shanshui Zeng ${ }^{1}$, Mengru Han ${ }^{1}$, Junjie Qin ${ }^{2}$, \\ Shenghui $\mathrm{Li}^{2}$, Shaochuan $\mathrm{Li}^{4 *}$ and Hongling Yang ${ }^{1 *}$ \\ ${ }^{1}$ Department of Laboratory, Guangzhou Women and Children's Medical Centre, Guangzhou Medical University, Guangzhou, \\ China, ${ }^{2}$ Shenzhen Promegene Technology Co., Ltd, Shenzhen, China, ${ }^{3}$ School of Public Health, Guangzhou Medical \\ University, Guangzhou, China, ${ }^{4}$ School of Biology and Biological Engineering, South China University of Technology, \\ Guangzhou, China
}

\section{OPEN ACCESS}

Edited by:

Marloes Dekker Nitert, The University of Queensland, Australia

Reviewed by: Junguk Hur, University of North Dakota, United States Mingbang Wang, Fudan University, China

*Correspondence: Hongling Yang hlyang62@163.com

Shaochuan Li stephen.li1126@gmail.com

${ }^{\dagger}$ These authors have contributed equally to this work

Specialty section:

This article was submitted to Microbiome in Health and Disease,

a section of the journal Frontiers in Cellular and Infection Microbiology

Received: 09 April 2020 Accepted: 06 January 2021 Published: 25 February 2021

Citation: Long Y, Liang F, Guo R, Zhu C, Zhao $X$, Wang $X$, Liu $F$, Jiang $M$, Liang Q, Zeng S, Han M, Qin J, Li S, Li S and Yang H (2021) Gut Microbiota Signatures in Gestational Anemia. Front. Cell. Infect. Microbiol. 11:549678.

doi: 10.3389/fcimb.2021.549678
Gestational diseases are associated with altered intestinal microbiota in pregnant women. Characterizing the gut microbiota of gestational anemia (GA) may describe a novel role of gut microbial abnormality in GA. In this study, we investigated differences in gut microbiota between GA patients and healthy pregnant women from the first trimester ( $n=24$ vs. 54) and the third trimester ( $n=30$ vs. 56) based on the 16S rRNA gene sequencing method. No statistically significant differences in $\alpha$-diversity were identified between GA patients and controls in the first trimester of pregnancy, whereas the Shannon index and observed OTUs were significantly lower in GA patients than in healthy controls in the third trimester. Distance-based redundancy analysis revealed striking differences in microbial communities in the third trimester between GA patients and controls. Four genera were significantly different in relative abundance between GA patients and healthy controls, while 12 genera differentiated significantly between GA patients and healthy controls in the third trimester. At the operational taxonomic unit (OTU) level, 17 OTUs and 30 OTUs were identified to be different between GA patients and healthy controls in the first and third trimesters, respectively. Changes in gut microbial composition of GA patients suggest a potential relation with $\mathrm{GA}$, and provide insights into the prediction and intervention of gestational anemia.

Keywords: gut microbiota, microbial dysbiosis, 16S rRNA gene sequencing, pregnant women, gestational anemia

\section{INTRODUCTION}

Anemia remains a major health problem worldwide, especially in third-world countries (Milman, 2011). The global anemia prevalence was estimated to be approximately $33 \%$ in 2010 (Kassebaum et al., 2014). Specifically, gestational anemia (GA), which occurs in pregnant women, is one of the most common types of anemia (W. H. Organization, 1992). According to the World Health Organization, the prevalence of GA was more than $40 \%$ in 2011 (World Health Organization, 2019). A crosssectional study showed that GA prevalence reached $58.6 \%$ in the third trimester of pregnancy for women in China (Ma et al., 2009). Anemia in pregnancy can impose a heavy physiological and 
economic burden on patients. GA may significantly affect the health status of both mothers and their fetuses (Ma et al., 2009). Previous studies have indicated that gestational anemia may increase the risk of intrauterine growth restriction, preterm birth, and low birth weight (Katz et al., 2006; Lee et al., 2006). Severe anemia can also increase perinatal morbidity (Prema et al., 1981) and lead to maternal death (Starrs, 2015).

There are a variety of causes of anemia. Nutritional deficiency of iron may result in development of iron deficiency anemia (IDA), which leads to a total burden of about 75 to $80 \%$ of anemia cases (Milman, 2011). Similarity, a lack of nutritional elements such as folate (Al Khatib et al., 2006; de Benoist, 2008), vitamin B12 (Pinto et al., 1973), and vitamin D (Özsoylu and Aytekin, 2011) can also contribute to anemia. Moreover, microbe invasion is also another important pathogenic factor for anemia; for example, malaria parasite can lead to sequestration in the placental vascular space with consequent maternal anemia (Shulman et al., 1996; Cot et al., 1998).

In recent years, there has been emerging evidence indicating the role of gut microbiota in human nutrition and metabolism. Studies in iron-deficient women from India indicated that the intestinal microbiota of individuals with IDA are relatively deficient in lactobacillus (Balamurugan et al., 2010). Another study reported gut microbiological disorders in infants and young children with nutritional IDA (Muleviciene et al., 2018), based on $16 \mathrm{~S}$ analyses of fecal samples. Moreover, gut microbiota can promote hematopoiesis at primary immune sites of the host (Khosravi et al., 2014), indicating the essential role of the gut microbiome in anemia. In addition to human nutrition, a study revealed that changes of gut microbiota may impact the circulating levels of short-chain fatty acids (SCFAs), further influence bone marrow hematopoiesis and the progress of infection (Jaeggi et al., 2015).

Pregnant women with GA comprise one of the most vulnerable cohorts of patients suffering from anemia. However, the alteration of gut microbiota and the association between the altered bacterial taxa and GA disease in pregnant women remains poorly understood. In this study, we explored the characteristics of gut microbial composition in 54 GA pregnant women in the first and third trimesters compared to 110 age- and body-weight-matched controls using $16 \mathrm{~S}$ rRNA gene sequencing.

\section{MATERIALS AND METHODS}

\section{Ethics Statement}

This study received approval from the Ethics Committee of Guangzhou Women and Children's Medical Centre, and informed consent was obtained from each subject. These methods were carried out in accordance with the Declaration of Helsinki (World Medical Association Declaration of Helsinki, 2014).

\section{Study Design and Fecal Sample Collection}

The matched case-control study recruited participants from Guangzhou Women and Children's Medical Centre from January 2017 to December 2017. Enrolled patients included 24 women with gestational anemia (GA1 group) and 54 gender-, age-, and body-weight-matched healthy pregnant women ( $\mathrm{HC} 1$ group) in the first trimester, as well as 30 women with gestational anemia (GA3 group) and 56 healthy controls (HC3 group) in the third trimester. In the present study, the inclusive criterion for gestational anemia was considered to be serum hemoglobin (HGB) $\leq 105 \mathrm{~g} / \mathrm{L}$, while the healthy controls were defined to be HGB $\geq 120 \mathrm{~g} / \mathrm{L}$. The phenotypic characteristics of all participants are summarized in Table 1. All participants were Chinese and no participants with alcoholism, smoking, strict vegetarians, or with other unusual dietary habits during pregnancy. The exclusion criteria included participants who had taken antibiotic treatment or probiotic supplements in the 4 weeks prior to sample collection. Baseline information was measured and fecal samples were collected by well-trained staff, strictly following standard procedures. Stool samples were put into a foam box filled with ice packs and transported to the laboratories as quickly as possible. Samples were stored at $-80^{\circ} \mathrm{C}$ until DNA extraction and $16 \mathrm{~S}$ rRNA gene sequencing. For all participants, iron supplementation intaking condition was conducted by questionnaire. No significant difference in iron supplement intake between GA group and HC group.

\section{DNA Extract and Sequencing}

Microbial DNA was extracted from stool samples collected by subjects in the hospital according to the MOBIO Power Soil ${ }^{\circledR}$ DNA Isolation Kit $12888-100$ protocol. All of the DNA was stored in a freezer at $-80^{\circ} \mathrm{C}$ before sequencing. Designed unique

TABLE 1 | Characteristics of the subjects.

\begin{tabular}{|c|c|c|c|c|}
\hline & & GA patients & Healthy controls & $P$-value \\
\hline & No. of samples & 24 & 54 & \\
\hline \multirow[t]{5}{*}{ 1st trimester } & HGB (g/L) & $97.8 \pm 7.5$ & $128.8 \pm 5.8$ & $<0.001$ \\
\hline & Age (year) & $28.8 \pm 3.6$ & $30.0 \pm 3.4$ & 0.189 \\
\hline & PBMI $\left(\mathrm{kg} / \mathrm{m}^{2}\right)$ & $19.7 \pm 2.1$ & $19.9 \pm 1.6$ & 0.667 \\
\hline & Gestational days & $100.9 \pm 6.5$ & $102.3 \pm 7.0$ & 0.409 \\
\hline & No. of samples & 30 & 56 & \\
\hline \multirow[t]{4}{*}{ 3rd trimester } & HGB (g/L) & $99.0 \pm 4.7$ & $125.9 \pm 5.8$ & $<0.001$ \\
\hline & Age (year) & $31.2 \pm 3.9$ & $30.8 \pm 3.6$ & 0.624 \\
\hline & PBMI $\left(\mathrm{kg} / \mathrm{m}^{2}\right)$ & $20.3 \pm 2.0$ & $20.6 \pm 2.1$ & 0.410 \\
\hline & Gestational days & $236.8 \pm 8.0$ & $235.6 \pm 7.9$ & 0.513 \\
\hline
\end{tabular}

The data for GA patients and healthy controls are presented as the mean \pm SD. P-values were calculated by Student's t-test. HGB, hemoglobin; PBMI, pre-pregnant body mass index. 
fusion primers with universal primers set, 515F (5' -GTGYCA GCMGCCGCGGTAA-3') and 806R (5' -GGACTACNVGGG TWTCTAAT- $3^{\prime}$ ), along with barcode sequence were used to amplify the V4 region of $16 \mathrm{~S}$ rRNA gene. PCR mixtures and thermal cycling were performed as previously described (Li et al., 2019). Amplicons from each sample were run on an agarose gel. The expected band size is $\sim 300-350 \mathrm{bp}$. Amplicons were quantified with the Quant-iT PicoGreen dsDNA Assay Kit (ThermoFisher/Invitrogen cat. no. P11496; following the manufacturer's instructions).

The amplicon library was pooled in equal amount and subsequently quantified (KAPA Library Quantification Kit KK4824). Then paired-end sequencing on Illumina MiniSeq platform at Promegene Co. Ltd (Shenzhen, China) was performed. Read length is 150 bp excluding the primer sequences.

\section{Bioinformatic Analyses}

Raw sequencing reads, which produced $>8$ homopolymers, $>2$ mismatches in the primers, or $>1$ mismatches in the barcode, were removed in pairs. High-quality sequencing reads were analyzed via the quantitative insights into microbial ecology (QIIME2, https://qiime2.org/) platform (Kuczynski et al., 2012) and the standard tools/plugins provided by QIIME2. Briefly, the $16 \mathrm{~S}$ sequences were analyzed for further quality control and to feature table construction using the DADA2 algorithm (Callahan et al., 2016). The remaining reads were truncated from 0 to 140 bases (for both forward and reverse reads) to avoid sequencing errors at the end of the reads. Paired-end reads were overlapped at the maximum mismatch of six bases, which created a minimum similarity threshold of approximately $90 \%$ on the overlap zone of the forward and reverse reads. The representative sequences (named "feature" in QIIME2 nomenclature) were then generated by removing the redundant and low occurrence ( $\mathrm{n}<5$ in pool samples) sequences. We used the term "operational taxonomic unit (OTU)" instead of "feature" throughout this article for convenience. Then, taxonomic assignment of the OTUs was determined based on a pretrained Naive Bayes classifier (DeSantis et al., 2006) (trained on the Greengenes 13_8 99\% OTUs) via the q2-feature-classifier plugin. The taxonomic compositions at the phylum, class, order, family, genus, and species levels were generated based on OTU annotation. To avoid sampling depth bias, 20,000 reads were randomly selected from each sample when calculating the OTU and taxa relative abundances.

Phylogenetic analyses were implemented via the $\mathrm{q} 2$ phylogeny plugin, which performed multiple sequence alignments on the OTU sequences and generated phylogenetic trees of the OTUs from the alignment results. Four estimators of the $\alpha$-diversity, including Shannon's diversity index, observed OTUs, Faith's phylogenetic diversity (a qualitative measure of community richness that incorporates the phylogenetic relationships between the OTUs) and Pielou's evenness, and Bray-Curtis dissimilarity (an estimator of the $\beta$-diversity) was used in this study and calculated based on the QIIME2 q2diversity plugin.

\section{Statistical Analyses}

Statistical analyses were implemented using the $\mathrm{R}$ platform. Distance-based redundancy analysis (dbRDA) was performed on normalized taxa abundance matrices with $\mathrm{R}$ vegan package (Dixon, 2003) according to Bray-Curtis dissimilarity, and then visualized with R ggplot2 package. The disease-associated genera, OTUs, and taxa were identified based on the Wilcoxon rank-sum test. Random forest models were trained with $\mathrm{R}$ randomForest package $(10,000$ trees $)$ to predict disease status according to OTU and genus abundance profiles. The performance of the predictive model was evaluated with leave-one-out crossvalidation method. Receiver operator characteristic (ROC) analysis was performed using $\mathrm{R}$ pROC package. A P-value of $<0.05$ was considered statistically significant, and the q-value was calculated to evaluate the false discovery rate for correction of multiple comparisons.

\section{RESULTS}

\section{Study Cohort and Sequencing Data}

To investigate the associations between the gut microbial composition and gestational anemia in pregnancy, we analyzed the fecal samples of healthy controls and women with GA in the first $(\mathrm{n}=24$ vs. 54) and third $(\mathrm{n}=30$ vs. 56) trimesters. According with the meta-analysis (Smith et al., 2019; Rahmati et al., 2020), the first and the third trimester have greater impact on pregnancy outcome, for example, anemia at the first and the third trimesters is associated with preterm birth and low birth weight. Therefore, we enrolled the pregnant women in the first trimester and the third trimester, which refers to the method of sample collection in some articles (Koren et al., 2012; NurielOhayon et al., 2019). Patients and controls were matched according three indicators (age, BMI, and gestational age) in each trimester (Table 1), and the first and third trimester cohorts are independent of each other without any overlap. Basic information of participants is shown at Table $\mathbf{1}$.

The gut microbiota from 164 fecal samples was profiled using high throughput 16S rRNA gene sequencing of the V4 variable region. A total of $8,539,948$ high quality sequences $(52,073 \pm$ 11,821 sequences per sample) were ultimately produced. And 2,453 OTUs were identified and functionally labeled using QIIME2 platform, as previously discussed.

\section{Reduced Diversity in Gestational Anemia in the Third Trimester}

The microbial $\alpha$ - and $\beta$-diversity indices were used to evaluate the richness and inter-sample relationships of gut microbiota of all participants. No statistically significant differences in $\alpha$ diversity were found between patients and controls in the first trimester of pregnancy (Figure 1A). However, when we focused on the third trimester of pregnancy, Shannon's diversity index and observed OTUs of gut microbiota in the GA group were significantly lower than those in the control group. The other three indices-Pielou's evenness, Faith's phylogenetic diversity index, and the observed OTUs diversity index-were lower in tendency. Furthermore, distance-based redundancy analysis 

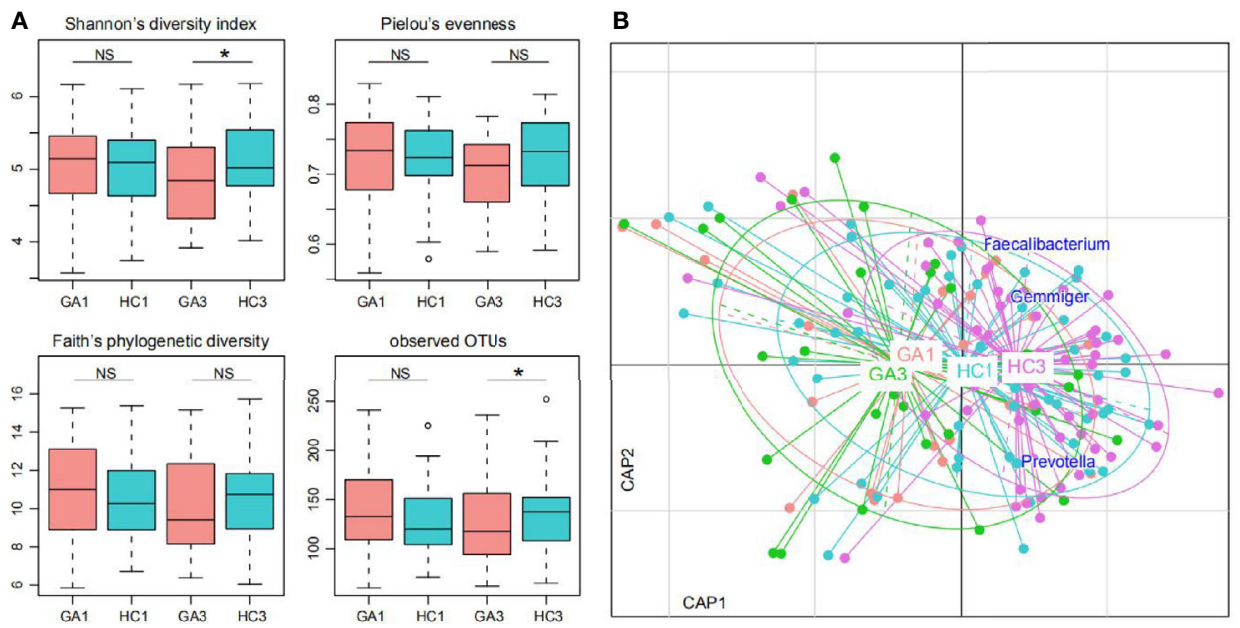

FIGURE 1 | Difference of gut microbial community between GA patients and controls. (A) Difference of $\alpha$-diversity between GA patients and controls. Significance levels in correlation tests are denoted: NS, P > 0.05; ${ }^{*} \mathrm{P} \leq 0.05$. (B) dbRDA based on the Bray-Curtis dissimilarity between microbial genera, revealing GA microbial dysbiosis, which overlaps only in part with taxonomic composition in patients and controls. Patient and control samples were mainly separated in the primary constrained axis. Lines connect samples (colored points) in the same group, and circles cover samples near the center of gravity for each group. Genera (blue squares) as the main contributors are plotted by their loadings in these two components.

( $\mathrm{dbRDA}$ ) based on Bray-Curtis dissimilarity between microbial genera captured visible separation of GA stratification on the intestinal microbiota when compared to the control group in both of the two time periods. However, the differences were not significant (Adonis $\mathrm{p}>0.05$ ) (Figure 1B). On the dbRDA plot, GA acted on the primary and the second constrained axis $(17.7 \%$ variance explained), while Prevotella, Faecalibacterium, and Gemmiger were the major contributors.

\section{Comparison of the Gut Microbiota}

At the phylum level, the predominant sequences in the GA1 were from Firmicutes (75\%), Bacteroidetes (17.2\%), Actinobacteria (5.5\%), Proteobacteria (1.6\%), and Verrucomicrobia (0.4\%), while the sequences for the $\mathrm{HC} 1$ group belonged to Firmicutes (80.3\%), Bacteroidetes (11.4\%), Actinobacteria (5\%), Proteobacteria (2.4\%), and Verrucomicrobia (0.6\%). These five phyla comprised more than $99 \%$ of the relative abundance in the two groups (Figure 2A). In the third trimester of pregnancy, Firmicutes was still the dominant phylum, which accounts for $75 \%$ abundance in GA3 and $81.4 \%$ in HC3; Bacteroidetes, Actinobacteria, Proteobacteria, and Euryarchaeota, contributed to $15,6.7,2.4$, and $0.2 \%$ abundance of the GA3 group and 9.7, $6.6,1.4$, and $0.4 \%$ abundance of the $\mathrm{HC} 3$ group, respectively (Figure 2A). The ratio of Firmicutes/Bacteroidetes in GA patients compared to controls expanded from 4.36 to 7.04 in the first trimester and 5.0 to 8.39 in the third trimester.

At the genus level, Turicibacter, Oribacterium, Parvimonas, and Anaerotruncus were significantly enriched in the GA1 group compared to the $\mathrm{HC1}$ group (Figure 2B), whereas no genus was significantly enriched in $\mathrm{HC1}$. In addition, eight genera, including Adlercreutzia, Gemmiger, Anaerostipes, Ruminococcaceae ${ }^{\star}$, Clostridiales ${ }^{\star}$, [Mogibacteriaceae] ${ }^{\star}$, Ruminococcus, and Faecalibacterium were more represented in the HC3 group
(Figure 2C), while Atopobium, Clostridiaceae ${ }^{*}$, Catenibacterium, and Eggerthella were enriched in the GA3 group compared to HC3 group.

After abundance filtering at an average relative abundance threshold of $0.01 \%, 483$ OTUs and 497 OTUs were obtained in the first and third trimesters, respectively. Six OTUs, including two each from family Ruminococcaceae, family Lachnospiraceae, species Veillonella dispar, and genus Oscillospira were enriched in GA patients in the first trimester compared with healthy controls. There were 11 OTUs that were depleted in GA patients in the first trimester, including genus Coprococcus, species Bacteroides uniformis, family Ruminococcaceae, species Clostridium celatum, genus Turicibacter, genus Bacteroides, o_Clostridiales, family Bacteroidales_S24-7, species Prevotella copri, genus Coprobacillus, and family Lachnospiraceae (Figure 3A). In the third trimester, 20 OTUs, such as species Gemmiger formicilis, species Faecalibacterium prausnitzii, species Bacteroides uniformis, species Coprococcus catus, genus Anaerostipes, genus Ruminococcus, genus Adlercreutzia, family [Mogibacteriaceae], and family Lachnospiraceae were identified to be more abundant in pregnant women with anemia. In contrast, another 10 OTUs, including three from genus Blautia, species [Ruminococcus] gnavus, species Prevotella copri, family Clostridiaceae, genus Streptococcus, genus Catenibacterium, family Lachnospiraceae, and o_Mollicutes_RF39, were enriched in pregnant women without anemia (Figure 3B). It is worth noting that genus Oscillospira were depleted in pregnant women without anemia in both trimesters.

\section{Gestational Anemia-Related Bacteria Are Associated With Inflammation and Metabolism}

For GA-related bacteria in first trimester, family Lachnospiraceae and species Prevotella_copri are negatively correlated with liver 

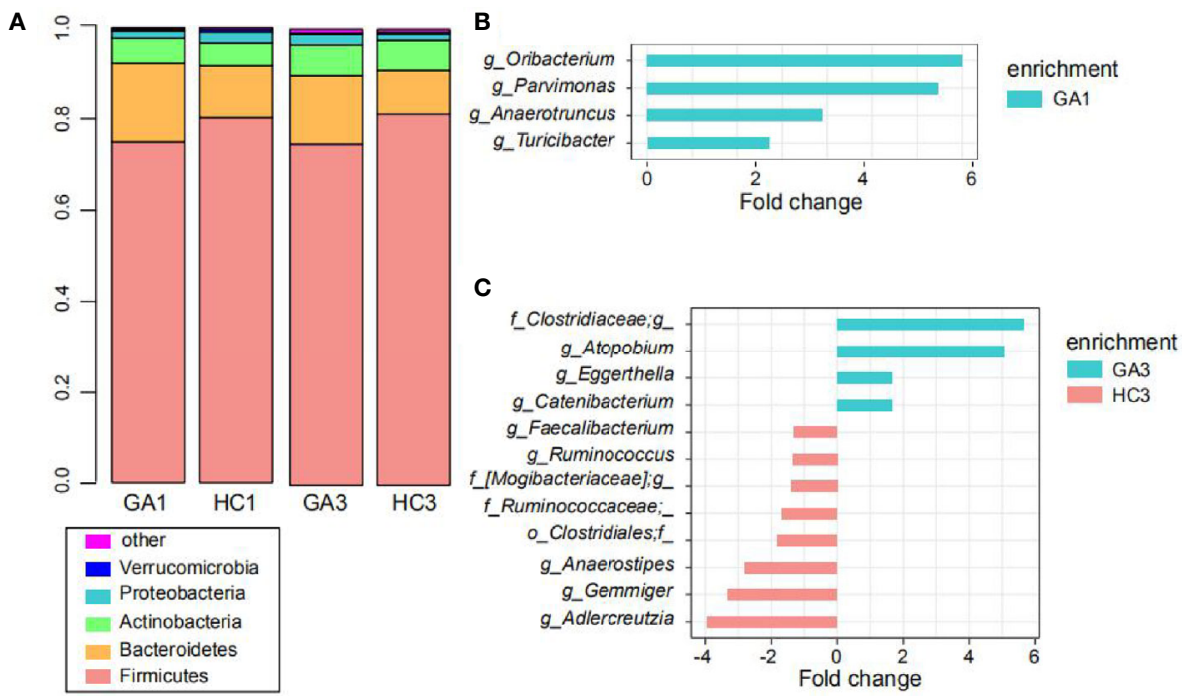

FIGURE 2 | Comparison of the gut microbiota. (A) Top five most abundant bacterial phyla in the GA patients and healthy controls in the first and third trimesters. Each colored box represents a bacterial taxon and the height of a colored box represents the relative abundance of that organism within the sample. Bacteria ranked below fifth, as well as unclassified phyla, are grouped as "other." (B, C) Bacterial genera showing significant differences in relative abundance between the GA patients and healthy controls in the first $\mathbf{( B )}$ and third $(\mathbf{C})$ trimesters.
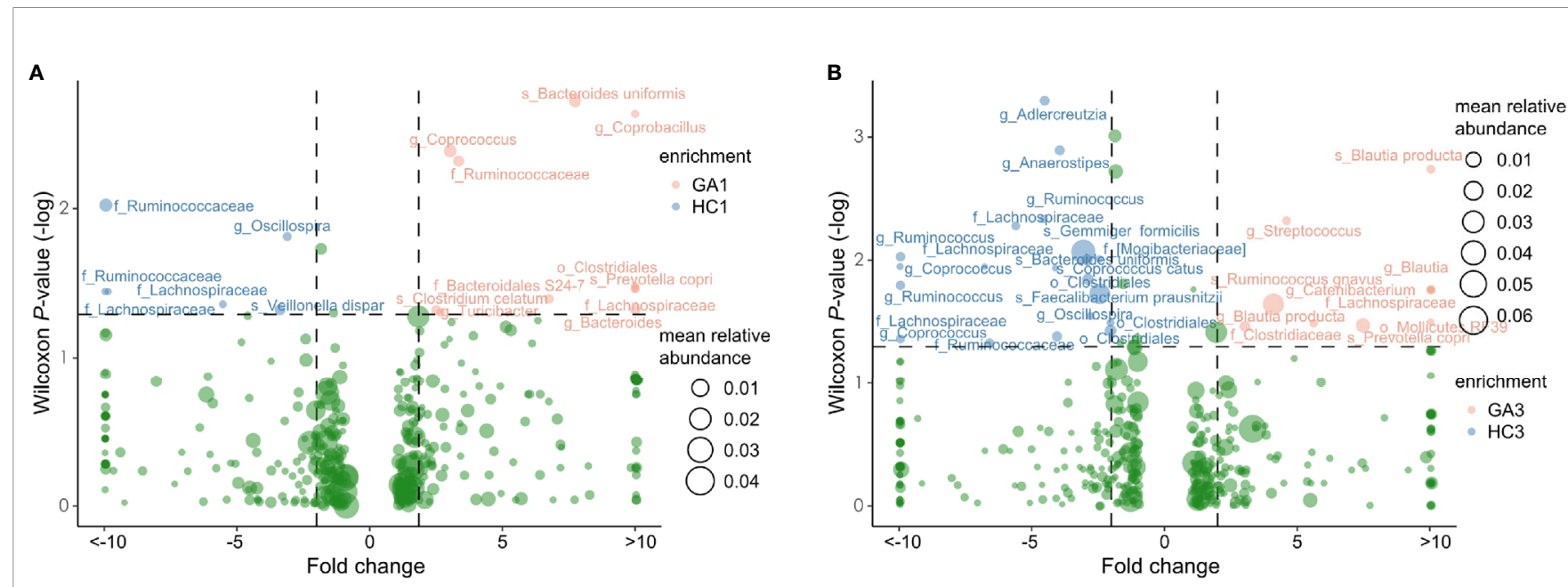

FIGURE 3 | Volcano plot showing significant variation in OTUs between patients and controls in the first (A) and third (B) trimesters. Only the OTUs with average relative abundances greater than $0.01 \%$ of total abundance in all samples are shown for clarity. Red and blue circles represent the GA- and control-enriched OTUs, respectively.

metabolism index, such as $\gamma$-GT, TP, ALB, TBA, GLO, ALT, AST, HDP, and BIL. Genus Coprococcus, family Lachnospiraceae, species Bacteroides uniformis, and family Ruminococcaceae are negatively correlated with inflammation index, such as BA, PCT, WBC, and EO, while species Prevotella copri and genus Coprococcus are positively correlated with BA (Figure S5A).

For GA-related bacteria in third trimester, genus Ruminococcus, species Bacteroides uniformis, genus Anaerostipes, order Clostridiales, species Faecalibacterium_prausnitzii, species Gemmiger formicilis, species Coprococcus catus, genus
Adlercreutzia, and family Lachnospiraceae are positively correlated with liver metabolism index (ALB and ALB/GLO), and inflammation index (WBC, $\mathrm{MCH}$, and $\mathrm{MCV}$ ), while species Ruminococcus gnavus and species Blautia producta are negatively correlated with liver ALB, $\mathrm{ALB} / \mathrm{GLO}, \mathrm{MCH}$, and MCV BA (Figure S5B).

\section{Gut Microbiota-Based Classification of Gestational Anemia}

Our study further provided we evaluated the ability of intestinal microbial composition to classify the anemic status of women. 
The models, trained from the abundance of OTU biomarkers in first and third trimesters, achieved the area under the ROC curve (AUC) of 0.80 (95\% CI 0.69 to 0.90 ) and 0.75 (95\% CI 0.63 to 0.84 ) for discriminating GA and healthy controls in the first or third trimester, respectively (Figures 4A, B, S1-S3). In addition, Blautia, Odoribacter, Ruminococcaceae ${ }^{*}$, Turicibacter, and Clostridiales ${ }^{*}$ featured the highest score in the model.

\section{DISCUSSION}

In the present study, we characterized the gut microbiota of pregnant women with anemia during at the first and third trimesters compared with those of healthy pregnant women. The results identified some differences in the composition of gut microbiota between anemia patients and normal controls.

In this study, although no significant differences in microbial $\alpha$-diversity were found between GA patients and healthy controls in the first trimester, a significantly reduced $\alpha$ diversity was found in GA patients during the third trimester. A reduction in $\alpha$-diversity in gut microbiota has also been described in other diseases, including Crohn's disease (Manichanh et al., 2006), atopic eczema (Wang et al., 2008), and myalgic encephalomyelitis/chronic fatigue syndrome (Giloteaux et al., 2016). The dbRDA analysis based on the Bray-Curtis distance (a $\beta$-diversity index) revealed that a common microbial feature emerged in our study. This indicates that the gut microbiota was significantly altered in GA patients. Changes in gut microbiota were reported to be associated with gestational diseases, such as gestational diabetes mellitus and early-onset preeclampsia (EOPE). Although the causes of gestational anemia remain elusive, our present study suggests that the composition of gut microbiota may be involved in the pathogenesis of GA.

The overall microbial composition of the fecal samples indicated that there were healthy adults in our study, with a dominance of taxa from the phyla of the Firmicutes, Bacteroidetes, and Actinobacteria (Curtis et al., 2012). It is worth noting that Firmicutes were less abundant in GA patients than in healthy controls, while Bacteroidetes were increased in GA patients compared to the healthy group in both trimesters. Moreover, the ratio of Firmicutes/Bacteroidetes, which may be positively correlated with obesity in healthy adults (Koliada et al., 2017), was higher in healthy controls than in GA patients. In agreement with this, thin women were more likely to be anemic than women of normal weight (Bentley and Griffiths, 2003).

At the genus level, Faecalibacterium was dominant in all four cohorts, and its abundance in GA patients was significantly lower than that in healthy controls in the third trimester. Consistently, at the OTU level, F. prausnitzii, which accounted for the majority of the abundance of Faecalibacterium, was also significantly decreased in GA patients in the third trimester. The importance of $F$. prausnitzii in the composition of gut microbiota has been demonstrated by many case control studies, such as colorectal cancer (Sobhani et al., 2011), liver transplantation (Wu et al., 2012), and chronic idiopathic diarrhea (Swidsinski et al., 2008). Decreased abundance of F. prausnitzii has been reported to be associated with dysbiosis caused from numerous disease (Miquel et al., 2013). For example, previous studies found that low levels of F. prausnitzii could be predictive for Crohn's disease (Sokol et al., 2008), and another study indicated that patients with Celiac disease have a significant decrease in the relative abundance of F. prausnitzii (De Palma et al., 2010).

Similarly, the relative abundance of Oscillospira (F0167), which was thought to be negatively associated with inflammatory diseases and body mass index (Konikoff and Gophna, 2016), was significantly higher in the healthy group than in the GA group during both trimesters. Oscillospira was decreased in patients or relatively severe patients in two studies of inflammatory bowel disease (Santoru et al., 2017) and human
A

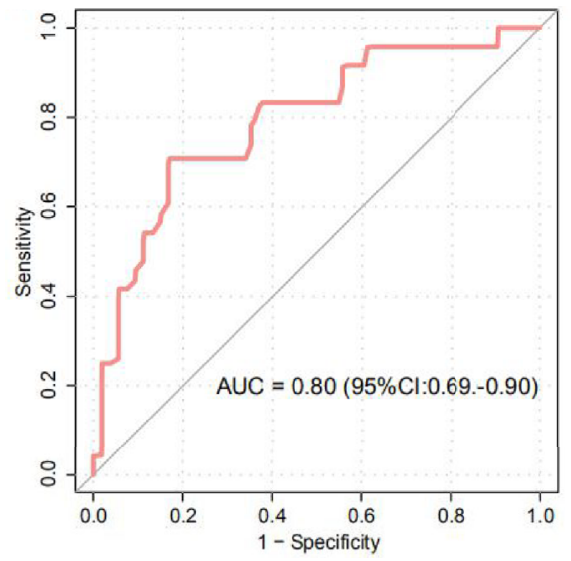

B

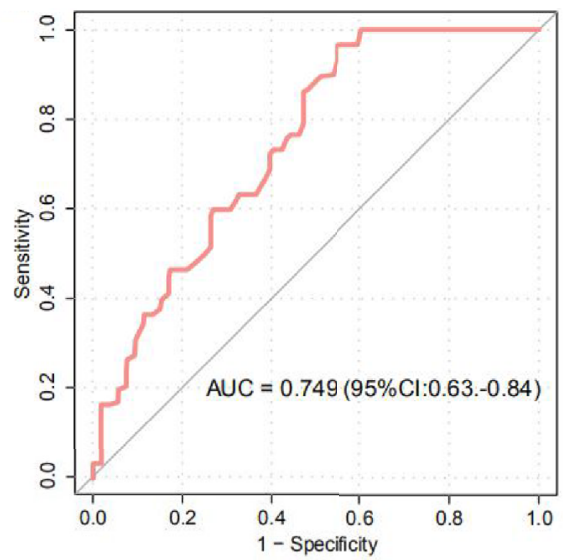

FIGURE 4 | Random Forest Classification of GA status by the abundance of gut microbiota. (A, B) ROC analysis for classification of GA status in the first (A) and third (B) trimesters by significantly different OTUs, assessed by AUC. 
immunodeficiency virus infection (Vesterbacka et al., 2017). Coincident results emerged in another genus of Ruminococcus, which was more abundant in healthy controls than in GA patients in the third trimester. It is not difficult to find that both Faecalibacterium and Ruminococcus are considered to be butyrate-producing members in human gut (Takahashi et al., 2016), and some of Oscillospira may also have the ability to produce butyrate (Gophna et al., 2017). Existing research indicated that butyrate plays a crucial role in the prevention of inflammation (Cushing et al., 2015) and metabolic diseases (Qin et al., 2012; Karlsson et al., 2013; Vrieze et al., 2014; Arora and Backhed, 2016).

Our study further provided the microbial markers for GA discrimination, and achieved AUC of 0.80 and 0.75 for identifying disease status in the first and third trimesters, respectively. Future systematic investigations of these OTU and gene markers that featured the highest score for the discrimination of disease status would be of value. However, to better explore a good potential for prediction and early diagnosis of GA from the fecal microbiota in the first trimester for the third trimester, a longitudinal study needs to be performed where fecal samples from early pregnancy (non-anemic at that time) of pregnant women who develop anemia at late pregnancy in order to avoid the two cohorts (first and third trimesters) are independent of each other.

This study is not without limitations. Although our samples were age- and BMI-matched, other environmental and behavioral factors, such as geography (Kumar et al., 2016), lifestyle (Conlon and Bird, 2014), diet (Paola et al., 2010), and drug usage (Panda et al., 2014) may have contributed to the gut microbial community of GA patients. Moreover, this was a crosssectional study with a relatively small number of participants, and thus, large-scale cohort studies containing multiple types of GA are needed to confirm the results.

To our knowledge, this is the first study to explore the relationship between gut microbiota and gestational anemia in both the first and the third trimesters of pregnancy. We demonstrated that GA patients may suffer from microbiota dysbiosis, which is mainly manifested by lower diversity and changes in some microbial taxa compared with healthy controls. Our findings on potential biomarkers not only extend the knowledge of the etiology of anemia in pregnant women, but also provide insights into the prediction and intervention of anemia and gestational anemia.

\section{REFERENCES}

Al Khatib, L., Obeid, O., Sibai, A. M., Batal, M., Adra, N., and Hwalla, N. (2006). Folate deficiency is associated with nutritional anaemia in Lebanese women of childbearing age. Public Health Nutr. 9 (7), 921-927. doi: 10.1017/ PHN2005921

Arora, T., and Backhed, F. (2016). The gut microbiota and metabolic disease: current understanding and future perspectives. J. Intern. Med. 280 (4), 339349. doi: 10.1111/joim. 12508

Balamurugan, R., Mary, R. R., Chittaranjan, S., Jancy, H., Shobana Devi, R., and Ramakrishna, B. S. (2010). Low levels of faecal lactobacilli in women with irondeficiency anaemia in south India. Br. J. Nutr. 104 (7), 931-934. doi: 10.1017/ s0007114510001637

\section{DATA AVAILABILITY STATEMENT}

The raw sequencing dataset analyzed in this study has been deposited in the European Bioinformatics Institute (EBI) database under the accession code PRJEB31743 (https://www. ebi.ac.uk/ena/data/view/PRJEB31743). The OTU and taxonomic composition data, and the statistical scripts are available from the corresponding authors upon reasonable request.

\section{ETHICS STATEMENT}

The studies involving human participants were reviewed and approved by Ethics Committee of Guangdong Women and Children Hospital. The patients/participants provided their written informed consent to participate in this study.

\section{AUTHOR CONTRIBUTIONS}

HY, SHL, and SCL conceived and directed the study. FaL and RG did the analysis and visualization. XZ, FeL, MJ, QL, SZ, and MH collected the samples. YL and FaL drafted the manuscript. XW, $\mathrm{CZ}$, and JQ revised the manuscript. All authors contributed to the article and approved the submitted version.

\section{FUNDING}

This study was funded by the National Natural Science Foundation of China (NSFC31570116, 81871716), the Natural Science Fund of Guangdong Province (2018A0303130314), the Science and Technology Fund of Guangzhou (201707010019, 201707010182), and the Shenzhen Science and Technology Innovation Committee (CYZZ2017331174025983), and the financial support of National Key R\&D Program of China (2017YFD0400301).

\section{SUPPLEMENTARY MATERIAL}

The Supplementary Material for this article can be found online at: https:/www.frontiersin.org/articles/10.3389/fcimb.2021.549678/ full\#supplementary-material

Bentley, M. E., and Griffiths, P. L. (2003). The burden of anemia among women in India. J. Eur. J. Clin. Nutr. 57 (1), 52-60. doi: 10.1038/sj.ejcn.1601504

Callahan, B. J., McMurdie, P. J., Rosen, M. J., Han, A. W., Johnson, A. J., and Holmes, S. P. (2016). DADA2: High-resolution sample inference from Illumina amplicon data. Nat. Methods 13 (7), 581-583. doi: 10.1038/nmeth.3869

Conlon, M. A., and Bird, A. R. (2014). The impact of diet and lifestyle on gut microbiota and human health. Nutrients 7 (1), 17-44. doi: 10.3390/nu7010017

Cot, M., le Hesran, J. Y., Miailhes, P., Roisin, A., Fievet, N., Barro, D., et al. (1998). Effect of chloroquine prophylaxis during pregnancy on maternal haematocrit. Ann. Trop. Med. Parasitol. 92 (1), 37-43. doi: 10.1080/00034989860157

Curtis, H., Dirk, G., Rob, K., Sahar, A., Jonathan, H. B., Asif, T. C., et al. (2012). Structure, function and diversity of the healthy human microbiome. Nature 486 (7402), 207-214. doi: 10.1038/nature11234 
Cushing, K., Alvarado, D. M., and Ciorba, M. A. (2015). Butyrate and Mucosal Inflammation: New Scientific Evidence Supports Clinical Observation. Clin. Transl. Gastroenterol. 6, e108. doi: 10.1038/ctg.2015.34

de Benoist, B. (2008). Conclusions of a WHO Technical Consultation on folate and vitamin B12 deficiencies. Food Nutr. Bull. 29 (2 Suppl), S238-S244. doi: $10.1177 / 15648265080292$ s129

De Palma, G., Nadal, I., Medina, M., Donat, E., Ribes-Koninckx, C., Calabuig, M., et al. (2010). Intestinal dysbiosis and reduced immunoglobulin-coated bacteria associated with coeliac disease in children. BMC Microbiol. 10, 63. doi: $10.1186 / 1471-2180-10-63$

DeSantis, T. Z., Hugenholtz, P., Larsen, N., Rojas, M., Brodie, E. L., Keller, K., et al. (2006). Greengenes, a chimera-checked 16S rRNA gene database and workbench compatible with ARB. Appl. Environ. Microbiol. 72 (7), 50695072. doi: 10.1128/AEM.03006-05

Dixon, P. (2003). VEGAN, a package of R functions for community ecology. J. Veg. Sci. 14, 927-930. doi: 10.1111/j.1654-1103.2003.tb02228.x

Giloteaux, L., Goodrich, J. K., Walters, W. A., Levine, S. M., Ley, R. E., and Hanson, M. R. (2016). Reduced diversity and altered composition of the gut microbiome in individuals with myalgic encephalomyelitis/chronic fatigue syndrome. Microbiome 4 (1), 30. doi: 10.1186/s40168-016-0171-4

Gophna, U., Konikoff, T., and Nielsen, H. B. (2017). Oscillospira and related bacteria - From metagenomic species to metabolic features. Environ. Microbiol. 19 (3), 835-841. doi: 10.1111/1462-2920.13658

Jaeggi, T., Kortman, G. A., Moretti, D., Chassard, C., Holding, P., Dostal, A., et al. (2015). Iron fortification adversely affects the gut microbiome, increases pathogen abundance and induces intestinal inflammation in Kenyan infants. Gut 64 (5), 731-742. doi: 10.1136/gutjnl-2014-307720

Karlsson, F. H., Tremaroli, V., Nookaew, I., Bergstrom, G., Behre, C. J., Fagerberg, B., et al. (2013). Gut metagenome in European women with normal, impaired and diabetic glucose control. Nature 498 (7452), 99-103. doi: 10.1038/nature12198

Kassebaum, N. J., Jasrasaria, R., Naghavi, M., Wulf, S. K., Johns, N., Lozano, R., et al. (2014). A systematic analysis of global anemia burden from 1990 to 2010 . Blood 123 (5), 615-624. doi: 10.1182/blood-2013-06-508325

Katz, J., Christian, P., Dominici, F., and Zeger, S. L. (2006). Treatment effects of maternal micronutrient supplementation vary by percentiles of the birth weight distribution in rural Nepal. J. Nutr. 136 (5), 1389-1394. doi: 10.1093/ jn/136.5.1389

Khosravi, A., Yanez, A., Price, J. G., Chow, A., Merad, M., Goodridge, H. S., et al. (2014). Gut microbiota promote hematopoiesis to control bacterial infection. Cell Host Microbe 15 (3), 374-381. doi: 10.1016/j.chom.2014.02.006

Koliada, A., Syzenko, G., Moseiko, V., Budovska, L., Puchkov, K., Perederiy, V., et al. (2017). Association between body mass index and Firmicutes/ Bacteroidetes ratio in an adult Ukrainian population. BMC Microbiol. 17 (1), 120. doi: 10.1186/s12866-017-1027-1

Konikoff, T., and Gophna, U. (2016). Oscillospira: a Central, Enigmatic Component of the Human Gut Microbiota. Trends Microbiol. 24 (7), 523524. doi: 10.1016/j.tim.2016.02.015

Koren, O., Goodrich, J. K., Cullender, T. C., Spor, A., Laitinen, K., Bäckhed, H. K., et al. (2012). Host remodeling of the gut microbiome and metabolic changes during pregnancy. Cell 150 (3), 470-480. doi: 10.1016/j.cell.2012.07.008

Kuczynski, J., Stombaugh, J., Walters, W. A., Gonzalez, A., Caporaso, J. G., and Knight, R. (2012). Using QIIME to analyze 16S rRNA gene sequences from microbial communities. Curr. Protoc. Microbiol. 14, 927-930. doi: 10.1002/ 9780471729259.mc01e05s27. Chapter 1, Unit 1E.5.

Kumar, H., Du, T. E., Kulkarni, A., Aakko, J., Linderborg, K. M., Zhang, Y., et al. (2016). Distinct Patterns in Human Milk Microbiota and Fatty Acid Profiles Across Specific Geographic Locations. Front. Microbiol. 7, e21313, 1619. doi: 10.3389/fmicb.2016.01619

Li, J. L., Sheng, H., Li, Shao, C., Zhi, C. Z., Hong, L. D., Cheng, T., et al. (2019). Early-onset preeclampsia is associated with gut microbial alterations in antepartum and postpartum women. Front. Cell. Infect. Microbiol. 9, 224. doi: $10.3389 /$ fcimb.2019.00224

Lee, H. S., Kim, M. S., Kim, M. H., Kim, Y. J., and Kim, W. Y. (2006). Iron status and its association with pregnancy outcome in Korean pregnant women. Eur. J. Clin. Nutr. 60 (9), 1130-1135. doi: 10.1038/sj.ejcn.1602429

Ma, A. G., Schouten, E., Wang, Y., Xu, R. X., Zheng, M. C., Li, Y., et al. (2009). Anemia prevalence among pregnant women and birth weight in five areas in China. Med. Princ. Pract. 18 (5), 368-372. doi: 10.1159/000226290
Manichanh, C., Rigottier-Gois, L., Bonnaud, E., Gloux, K., Pelletier, E., Frangeul, L., et al. (2006). Reduced diversity of faecal microbiota in Crohn's disease revealed by a metagenomic approach. Gut 55 (2), 205-211. doi: 10.1136/gut. 2005.073817

Milman, N. (2011). Anemia-still a major health problem in many parts of the world! Ann. Hematol. 90 (4), 369-377. doi: 10.1007/s00277-010-1144-5

Miquel, S., Martin, R., Rossi, O., Bermudez-Humaran, L. G., Chatel, J. M., Sokol, H., et al. (2013). Faecalibacterium prausnitzii and human intestinal health. Curr. Opin. Microbiol. 16 (3), 255-261. doi: 10.1016/j.mib.2013.06.003

Muleviciene, A., D’Amico, F., Turroni, S., Candela, M., and Jankauskiene, A. (2018). Iron deficiency anemia-related gut microbiota dysbiosis in infants and young children: A pilot study. Acta Microbiol. Immunol. Hung 65 (4), 551-564. doi: 10.1556/030.65.2018.045

Nuriel-Ohayon, M., Neuman, H., Ziv, O., Belogolovski, A., Barsheshet, Y., Bloch, N., et al. (2019). Progesterone Increases Bifidobacterium Relative Abundance during Late Pregnancy. Cell Rep. 27 (3), 730-736.e3. doi: 10.1016/j.celrep.2019. 03.075

Özsoylu, Ş, and Aytekin, M.N.J.A. (2011). Vitamin D deficiency and anemia. Front. Microbiol. 90, 6, 737-737. doi: 10.1007/s00277-010-1078-y

Panda, S., El khader, I., Casellas, F., Lopez Vivancos, J., Garcia Cors, M., Santiago, A., et al. (2014). Short-term effect of antibiotics on human gut microbiota. PloS One 9 (4), e95476. doi: 10.1371/journal.pone.0095476

Paola, M. D., Filippo, C. D., Cavalieri, D., Ramazzotti, M., Poullet, J. B., Massart, S., et al. (2010). PP90 Impact Of Diet In Shaping Gut Microbiota Revealed By A Comparative Study In Children From Europe And Rural Africa. Proc. Natl. Acad. Sci. U.S.A. 107 (33), 14691-14696. doi: 10.1073/pnas.1005963107

Pinto, A. V., Santos, F., Almeida, A. M., and Cantuaria, A. A. (1973). Trends of folate and vitamin B12 during pregnancy. Rev. Invest. Clin. 25 (2), 153-158.

Prema, K., Neelakumari, S., and Ramalakshmi, B. A. (1981). Anaemia and adverse obstetric outcome.

Qin, J., Li, Y., Cai, Z., Li, S., Zhu, J., Zhang, F., et al. (2012). A metagenome-wide association study of gut microbiota in type 2 diabetes. Nature 490 (7418), 5560. doi: 10.1038/nature11450

Rahmati, S., Azami, M., Badfar, G., Parizad, N., and Sayehmiri, K. (2020). The relationship between maternal anemia during pregnancy with preterm birth: a systematic review and meta-analysis. J. Matern. Fetal Neonatal. Med. 33 (15), 2679-2689. doi: 10.1080/14767058.2018.1555811

Santoru, M. L., Piras, C., Murgia, A., Palmas, V., Camboni, T., Liggi, S., et al. (2017). Cross sectional evaluation of the gut-microbiome metabolome axis in an Italian cohort of IBD patients. Sci. Rep. 7 (1), 9523. doi: 10.1038/s41598-017-10034-5

Shulman, C. E., Graham, W. J., Jilo, H., Lowe, B. S., New, L., Obiero, J., et al. (1996). Malaria is an important cause of anaemia in primigravidae: evidence from a district hospital in coastal Kenya. Trans. R Soc. Trop. Med. Hyg. 90 (5), 535-539. doi: 10.1016/s0035-9203(96)90312-0

Smith, C., Teng, F., Branch, E., Chu, S., and Joseph, K. S. (2019). Maternal and Perinatal Morbidity and Mortality Associated With Anemia in Pregnancy. Obstet. Gynecol. 134 (6), 1234-1244. doi: 10.1097/aog.0000000000003557

Sobhani, I., Tap, J., Roudot-Thoraval, F., Roperch, J. P., Letulle, S., Langella, P., et al. (2011). Microbial dysbiosis in colorectal cancer (CRC) patients. PloS One 6 (1), e16393. doi: 10.1371/journal.pone.0016393

Sokol, H., Pigneur, B., Watterlot, L., Lakhdari, O., Bermudez-Humaran, L. G., Gratadoux, J. J., et al. (2008). Faecalibacterium prausnitzii is an antiinflammatory commensal bacterium identified by gut microbiota analysis of Crohn disease patients. Proc. Natl. Acad. Sci. U.S.A. 105 (43), 16731-16736. doi: $10.1073 /$ pnas.0804812105

Starrs, A. J. W. D. (2015). Preventing the Tragedy of Maternal Deaths. Front. Microbiol. 19, 5, 312. doi: 10.2307/1966803

Swidsinski, A., Loening-Baucke, V., Verstraelen, H., Osowska, S., and Doerffel, Y. (2008). Biostructure of fecal microbiota in healthy subjects and patients with chronic idiopathic diarrhea. Gastroenterology 135 (2), 568-579. doi: 10.1053/ j.gastro.2008.04.017

Takahashi, K., Nishida, A., Fujimoto, T., Fujii, M., Shioya, M., Imaeda, H., et al. (2016). Reduced Abundance of Butyrate-Producing Bacteria Species in the Fecal Microbial Community in Crohn's Disease. Digestion 93 (1), 59-65. doi: 10.1159/000441768

Vesterbacka, J., Rivera, J., Noyan, K., Parera, M., Neogi, U., Calle, M., et al. (2017). Richer gut microbiota with distinct metabolic profile in HIV infected Elite Controllers. Sci. Rep. 7 (1), 6269. doi: 10.1038/s41598-017-06675-1 
Vrieze, A., Out, C., Fuentes, S., Jonker, L., Reuling, I., Kootte, R. S., et al. (2014). Impact of oral vancomycin on gut microbiota, bile acid metabolism, and insulin sensitivity. J. Hepatol. 60 (4), 824-831. doi: 10.1016/j.jhep.2013.11.034

W. H. Organization (1992). The prevalence of anaemia in women: a tabulation of available information (Geneva: World Health Organization).

World Health Organization (2019). Assessment for nutrition-related disorders in women during pregnancy. Available at: https://www.who.int/elena/titles/ assessment-methods-pregnancy/en/.

Wang, M., Karlsson, C., Olsson, C., Adlerberth, I., Wold, A. E., Strachan, D. P., et al. (2008). Reduced diversity in the early fecal microbiota of infants with atopic eczema. J. Allergy Clin. Immunol. 121 (1), 129-134. doi: 10.1016/ j.jaci.2007.09.011

World Medical Association Declaration of Helsinki (2014). ethical principles for medical research involving human subjects. J. Am. Coll. Dent. 81 (3), 14-18. doi: 10.1515/jwiet-2014-0117

Wu, Z. W., Ling, Z. X., Lu, H. F., Zuo, J., Sheng, J. F., Zheng, S. S., et al. (2012). Changes of gut bacteria and immune parameters in liver transplant recipients.
Hepatobiliary Pancreat Dis. Int. 11 (1), 40-50. doi: 10.1016/S1499-3872(11) 60124-0

Conflict of Interest: Authors SHL, FaL, RG, XW, and JQ were employed by the company Shenzhen Promegene Technology Co., Ltd.

The remaining authors declare that the research was conducted in the absence of any commercial or financial relationships that could be construed as a potential conflict of interest.

Copyright (C) 2021 Long, Liang, Guo, Zhu, Zhao, Wang, Liu, Jiang, Liang, Zeng, Han, Qin, Li, Li and Yang. This is an open-access article distributed under the terms of the Creative Commons Attribution License (CC BY). The use, distribution or reproduction in other forums is permitted, provided the original author(s) and the copyright owner(s) are credited and that the original publication in this journal is cited, in accordance with accepted academic practice. No use, distribution or reproduction is permitted which does not comply with these terms. 